\title{
The Spleen as an Extrapulmonary Target of COVID-19.
}

\author{
Wesam Hassan ${ }^{1}$, Haidi Karam-Allah Ramadan' ${ }^{2}$ Ghada Ali Omran ${ }^{3}$ \\ ${ }^{1}$ Assiut Center for Viral Hepatitis Management, Ministry of Health, Assiut, Egypt. \\ ${ }^{2}$ Department of Tropical Medicine and Gastroenterology, Faculty of Medicine, \\ Assiut University, Assiut, Egypt. \\ ${ }^{3}$ Forensic Medicine and Clinical Toxicology Department, Faculty of Medicine, \\ Assiut university, Assiut, Egypt..
}

Corresponding Author Haidi Karam-Allah Ramadan

Mobile: $+201202330002$

E mail: haidikaram@aun.edu.e $g$

Key words: COVID-19; spleen; extrapulmonary
The spleen has been recently reported as one of the unusual extrapulmonary organs that can be affected by COVID-19. Different splenic lesions were described in COVID-19 patients. Splenic infarction was reported frequently, and ruptured spleen and splenic abscess were also reported. Splenic involvement in COVID-19 can be caused through direct virus damage of the spleen or COVID-19 induced microvascular thrombosis and vasculitis [1-7].

The hypercoagulable state induced by COVID-19 virus leads to splenic artery or vein thrombosis and splenic infarction development due to splenic hypoperfusion $[3,8]$. The management of splenic infarction is usually by heparin anticoagulation, however, with unstable cases splenectomy is life saving [3].

Pyogenic splenic abscess is a rare infection often occurs in an immunocompromised patient, management is usually performed by percutaneous drainage, antibiotics or splenectomy [9]. The splenic abscess in patients with COVID-19 may be attributed to necrosis of the spleen caused by direct attack of the virus [5]. Postmortem needle autopsies from the spleen of ten dead COVID19 patients reported by $\mathrm{Xu}$ et al., who showed varying degrees of $\mathrm{T}$ and $\mathrm{B}$ lymphocyte depletion, lymphoid follicles atrophy due to direct attack of the SARS-COV 2 virus as well [10].
Splenic rupture (SR) is a rare lifethreatening condition. Delay in diagnosis and management of SR can lead to fatal outcome. SR is mostly traumatic; however, it may complicate malignancies, hematological, inflammatory and infectious splenic diseases. The diagnosis of splenic rupture is confirmed by abdominal computed tomography scan (CT scan) or by surgical laparotomy in hemodynamically unstable patients. SR is usually managed by splenectomy; however, the spleen preserving procedures such as arterial embolization can be considered in stable patients to avoid overwhelming post-splenectomy infection. Rupture spleen in COVID- 19 may be related to direct damage to the spleen by the virus or secondary micro or macrovascular thromboembolism [6, 7].

Here, we summarize a few recent reports that have described splenic lesions in association with COVID-19 (Table 1) [1-7]. Splenic infarction was the most frequent pathology with COVID-19 that occurred in six patients, the majority of those patients were men; one patients was young age and had no comorbidities and severe abdominal pain was present in four patients. Other abdominal visceral infarctions in addition to splenic infarction was present in two patients; one had renal infarction and the other 
had intestinal ischemia. Hemoperitoneum and intraperitoneal collection were present in two patients. Treatment was by heparin anticoagulant; however, one case undergone splenectomy and resection of ischemic bowel loop [1-4].

The splenic abscess in COVID-19 was described in one man 55 years old with multiple comorbidities, CT abdomen confirmed pyogenic splenic abscess with impending rupture, pneumatosis intestinalis and pneumoperitoneum. The patient had undergone emergency splenectomy, however he died due to the development of multiorgan failure, severe sepsis and pulmonary embolism [5].

Notably, spontaneous splenic rupture occurred in two patients with COVID- 19, both were men, their age was above 50 years. Both patients had abdominal pain without clear evidence of bacteremia, one was shocked, that patient was managed by splenic artery embolization. Emergency surgical laparotomy and splenectomy were performed in the other patient. Both patients had hemoperitoneum, but eventually improved and discharged [6,7].

To conclude, the spleen may be an extrapulmonary target of COVID-19 virus and abdominal pain is alarming in COVID-19 infection, it may indicate a serious condition. CT abdomen is helpful in diagnosis of splenic complications secondary to COVID-19. Early diagnosis and intervention are life saving in most of these patients.

Declaration of interest: The authors declare that they have no conflict of interest to declare.

Funding: This research did not receive any specific grant from funding agencies in the public, commercial, or not-for-profit sectors.

\section{REFERENCES}

1- Pessoa MSL, Lima CFC, Pimentel ACF, Costa JCG. Multisystemic infarctions in COVID-19: focus on the spleen. European Journal of Case Reports in Internal Medicine 2020; 7.

2- Besutti G, Bonacini R, Iotti V, Marini G, Riva N, Dolci $\mathrm{G}$, et al. Abdominal visceral infarction in 3 patients with COVID-19. Emerging Infectious Diseases 2020; 26: 1926.

3- Qasim Agha O,Berryman R. Acute splenic artery thrombosis and infarction associated with COVID-19 disease. Case Reports in Critical Care 2020; 2020.

4- Karki S, Rawal SB, Malla S, Rayamajhi J, Thapa BB. A case report on spontaneous hemoperitoneum in COVID-19 patient. International Journal of Surgery Case Reports 2020; 75: 211-213.

5- Al-Ozaibi LS, Alshaikh MO, Makhdoom M, Alzoabi OM, Busharar HA, Keloth TR. Splenic Abscess: An Unusual Presentation of COVID19? Dubai Medical Journal 2020;3:115-118.

6- Shaukat I, Khan R, Diwakar L, Kemp T, Bodasing N. Atraumatic splenic rupture due to covid-19 infection. Clinical infection in practice 2020:100042.

7- Mobayen M, Yousefi S, Mousavi M, Anbaran AS. The presentation of spontaneous splenic rupture in a COVID-19 patient: a case report. BMC Surgery 2020; 20: 1-5.

8- Del Hoyo J, López-Muñoz P, Fernández-de la Varga M, Garrido-Marín A, Valero-Pérez E, Prieto M, et al. Hepatobiliary and Pancreatic: A fatal case of extensive splanchnic vein thrombosis in a patient with Covid-19. Journal of Gastroenterology and Hepatology 2020.

9- Davido B, Dinh A, Rouveix E, Crenn P, Hanslik $\mathrm{T}$, Salomon J. Abcès de la rate: du diagnostic au traitement. La revue de médecine interne 2017; 38:614-618.

10- Xu X, Chang X, Pan H, Su H, Huang B, Yang M, et al. Pathological changes of the spleen in ten patients with coronavirus disease 2019 (COVID19) by postmortem needle autopsy. Zhonghua bing li xue za zhi= Chinese journal of pathology 2020; 49:576-582. 
Table (1): Clinical characteristics and outcomes in COVID-19 patients with splenic lesions.

\begin{tabular}{|c|c|c|c|c|c|c|c|c|c|}
\hline & \multicolumn{2}{|c|}{ Pessoa et al [1] } & \multicolumn{2}{|c|}{ Bessutti et al [2] } & \multirow{2}{*}{\begin{tabular}{|c} 
Agha and \\
Berryman [3] \\
Case 5
\end{tabular}} & \multirow{2}{*}{$\begin{array}{c}\text { Karki et al } \\
\text { [4] } \\
\text { Case } 6\end{array}$} & \multirow{2}{*}{$\begin{array}{c}\text { Al-Ozaibi et al [5] } \\
\text { Case } 7\end{array}$} & \multirow{2}{*}{$\begin{array}{c}\text { Shaukat et al } \\
{[6]} \\
\text { Case } 8\end{array}$} & \multirow{2}{*}{$\begin{array}{c}\text { Mobayen et al [7] } \\
\text { Case } 9\end{array}$} \\
\hline & Case 1 & Case 2 & Case 3 & Case 4 & & & & & \\
\hline Splenic lesion & \begin{tabular}{|l|} 
Splenic \\
infarction
\end{tabular} & Splenic infarction & \begin{tabular}{|l} 
Splenic \\
infarction
\end{tabular} & Splenic infarction & $\begin{array}{l}\text { Splenic } \\
\text { infarction\& } \\
\text { Splenic artery } \\
\text { thrombosis }\end{array}$ & Splenic infarction & $\begin{array}{l}\text { Splenic } \\
\text { abscess }\end{array}$ & $\begin{array}{l}\text { Splenic } \\
\text { rupture }\end{array}$ & $\begin{array}{l}\text { Splenic } \\
\text { rupture }\end{array}$ \\
\hline Age, sex & 67, male & 53 , female & 53, male & 72, male & 60, male & 32 , male & 55, male & 57, male & 52, male \\
\hline Comorbidites & NA & $\begin{array}{l}\text { Rheumatoid } \\
\text { arthritis }\end{array}$ & $\begin{array}{l}\text { Hypertension, } \\
\text { Mitral valve } \\
\text { replacement }\end{array}$ & $\begin{array}{l}\text { Hypertension } \\
\text { DM, } \\
\text { Previous MI, } \\
\text { Stage } 3 \text { kidney } \\
\text { failure }\end{array}$ & $\begin{array}{l}\text { Hypertension } \\
\text { DM, } \\
\text { Morbid obesity, } \\
\text { IgG deficiency, } \\
\text { Asthma, } \\
\text { OSA }\end{array}$ & $\mathrm{NO}$ & \begin{tabular}{|l} 
DM \\
ischemic heart \\
disease, \\
chronic kidney \\
disease, \\
old \\
cerebrovascular \\
Stroke \\
\end{tabular} & OSA & NA \\
\hline \multicolumn{10}{|l|}{$\begin{array}{l}\begin{array}{l}\text { Clinical } \\
\text { presentation }\end{array} \\
\end{array}$} \\
\hline Fever & Yes & Yes & Yes & NO & Yes & Yes & Yes & NO & Yes \\
\hline Chest symptoms & $\begin{array}{l}\text { Cough, } \\
\text { dyspnea }\end{array}$ & $\begin{array}{l}\text { Cough, } \\
\text { dyspnea }\end{array}$ & $\begin{array}{l}\text { Cough, } \\
\text { sore throat }\end{array}$ & $\begin{array}{l}\text { Cough, } \\
\text { dyspnea }\end{array}$ & $\begin{array}{l}\text { Cough, } \\
\text { dyspnea }\end{array}$ & NO & NO & Cough & $\begin{array}{l}\text { Respiratory } \\
\text { distress }\end{array}$ \\
\hline $\begin{array}{l}\text { Abdominal } \\
\text { symptoms }\end{array}$ & NA & NA & $\begin{array}{l}\text { Severe } \\
\text { left flank } \\
\text { pain }\end{array}$ & $\begin{array}{l}\text { Severe } \\
\text { abdominal } \\
\text { Pain }\end{array}$ & $\begin{array}{l}\text { Severe } \\
\text { left upper } \\
\text { quadrant pain \& } \\
\text { diarrhea }\end{array}$ & $\begin{array}{l}\text { Moderate } \\
\text { to severe } \\
\text { Periumbilical } \\
\text { pain }\end{array}$ & $\begin{array}{l}\text { Acute } \\
\text { abdomen }\end{array}$ & $\begin{array}{l}\text { Acute } \\
\text { abdomen } \\
\text { \& anorexia } \\
\text { \& diarrhea }\end{array}$ & $\begin{array}{l}\text { Abdominal } \\
\text { Pain } \\
\text { \& nausea }\end{array}$ \\
\hline Others & $\begin{array}{l}\text { Weakness of left } \\
\text { upper limb, } \\
\text { drooping of } \\
\text { mouth, headache }\end{array}$ & Anosmia & NO & Metabolic acidosis & $\mathrm{NO}$ & Rash & $\begin{array}{l}\text { Aphasia, Right } \\
\text { sided } \\
\text { weakness, Sepsis }\end{array}$ & $\begin{array}{l}\text { Shocked } \\
\text { (Main } \\
\text { presentation) }\end{array}$ & NO \\
\hline CT chest & $\begin{array}{l}\text { Ground glass } \\
\text { opacities\& } \\
\text { pulmonary } \\
\text { thrombo- } \\
\text { embolism }\end{array}$ & $\begin{array}{l}\text { Ground glass } \\
\text { opacities }\end{array}$ & \begin{tabular}{|l} 
Bilateral \\
Viral \\
pneumonia
\end{tabular} & NA & $\begin{array}{l}\text { NA } \\
\text { Chest x-ray } \\
\text { show patchy } \\
\text { opacity in right } \\
\text { lung }\end{array}$ & $\mathrm{NA}$ & NA & \begin{tabular}{|l|} 
Ground \\
glass opacity and \\
consolidation
\end{tabular} & \begin{tabular}{|l|} 
Ground glass \\
and pleural \\
effusion \\
Extensive fluid \\
collection around \\
spleen
\end{tabular} \\
\hline
\end{tabular}

Hassan, Afro-Egypt J Infect Endem Dis 2021; 11(1):96-99

$$
\text { https://aeji.journals.ekb.eg/ }
$$

http://mis.zu.edu.eg/ajied/home.aspx 


\begin{tabular}{|c|c|c|c|c|c|c|c|c|c|}
\hline & \multicolumn{2}{|c|}{ Pessoa et al [1] } & \multicolumn{2}{|c|}{ Bessutti et al [2] } & \multirow{2}{*}{$\begin{array}{c}\text { Agha and } \\
\text { Berryman [3] } \\
\text { Case } 5\end{array}$} & \multirow{2}{*}{$\begin{array}{c}\text { Karki et al } \\
\text { [4] } \\
\text { Case } 6\end{array}$} & \multirow{2}{*}{\begin{tabular}{|c|} 
Al-Ozaibi et al [5] \\
Case 7
\end{tabular}} & \multirow{2}{*}{$\begin{array}{c}\text { Shaukat et al } \\
\text { [6] } \\
\text { Case } 8\end{array}$} & \multirow{2}{*}{$\begin{array}{c}\text { Mobayen et al [7] } \\
\text { Case } 9\end{array}$} \\
\hline & Case 1 & Case 2 & Case 3 & Case 4 & & & & & \\
\hline CT abdomen & Splenic infarction & $\begin{array}{l}\text { Splenic infarction, } \\
\text { filling defect in } \\
\text { the subsegmental } \\
\text { branches of } \\
\text { splenic artery }\end{array}$ & $\begin{array}{l}\text { Splenic infarction } \\
\& \\
\text { Left kidney } \\
\text { infarction }\end{array}$ & $\begin{array}{l}\text { Massive } \\
\text { splenic infarction } \\
\& \\
\text { Small bowel } \\
\text { ischemia }\end{array}$ & $\begin{array}{l}\text { More than } 50 \% \\
\text { splenic } \\
\text { infarction } \\
\& \\
\text { Acute splenic } \\
\text { artery } \\
\text { thrombosis }\end{array}$ & \begin{tabular}{|l} 
Splenic \\
infarction, \\
Hemo- \\
peritoneum
\end{tabular} & $\begin{array}{l}\text { Impending rupture } \\
\text { pyogenic splenic } \\
\text { Abscess } \\
\& \\
\text { Pneumo-peritoneum } \\
\& \\
\text { Colonic wall } \\
\text { thickening } \\
\text { with } \\
\text { Intramural gas }\end{array}$ & \begin{tabular}{|l|} 
Rupture spleen \\
$\&$ \\
Hemo-peritoneum
\end{tabular} & NA \\
\hline CBC & NA & NA & Normal & Normal & Normal & \begin{tabular}{|l|} 
Pancytopenia \\
Decreased HB \\
level from 14.1 to \\
$7.3 \mathrm{gm} \%$
\end{tabular} & WBC: $5 \times 10^{3} / \mathrm{mm}^{3}$ & $\begin{array}{l}\text { WBC:3.7 } \\
\left(\mathrm{x} 10^{3} / \mathrm{mm}^{3}\right) \\
\text { HB: } 7.8 \mathrm{~g} / \mathrm{dl} \\
\text { PLT:149 } \\
\left(\mathrm{x} 10^{3} / \mathrm{mm}^{3}\right)\end{array}$ & HB: $9.5 \mathrm{~g} / \mathrm{dl}$ \\
\hline INR & NA & NA & NA & Normal & NA & Normal & NA & Normal & Normal \\
\hline CRP $\mathrm{mg} / \mathrm{dl}$ & NA & NA & 4.4 & 48 & 86.6 & NA & 300.4 & 368.1 & NA \\
\hline D-dimer $\mathrm{ng} / \mathrm{ml}$ & NA & NA & NA & 6910 & 259 & Normal & NA & NA & NA \\
\hline Management & NA & NA & LMWH & $\begin{array}{l}\text { Splenectomy } \\
\& \\
\text { resection of } \\
\text { ischemic bowel } \\
\text { loop } \\
\end{array}$ & $\begin{array}{l}\text { Heparin drip for } \\
24 \mathrm{~h} \text { then shift to } \\
\text { Enoxaparin } \\
1 \mathrm{mg} / \mathrm{kg} \text { twice } \\
\text { daily }\end{array}$ & Supportive & $\begin{array}{l}\text { Emergent laprotomy } \\
\& \\
\text { splenectomy }\end{array}$ & $\begin{array}{l}\text { Splenic artery } \\
\text { embolization }\end{array}$ & $\begin{array}{l}\text { Emergent } \\
\text { laparotomy } \\
\& \text { splenectomy } \\
\text { was done }\end{array}$ \\
\hline Outcome & NA & NA & Improved & Improved & Improved & NA & $\begin{array}{l}\text { Died (severe sepsis, } \\
\text { multi organ failure, } \\
\text { PE) }\end{array}$ & Improved & Improved \\
\hline
\end{tabular}

DM: Diabetes mellitus, OSA: Obstructive sleep apnea, MI: myocardial infarction, PE: pulmonary embolism, LMWH: low molecular weight heparin, NA: Not available. 